\title{
A Comparison of Velocity Skin Effect Modeling with 2-D Transient and 3-D Quasi-transient Finite Element Methods
}

\author{
Nail Tosun (D), Graduate Student Member, IEEE, Doğa Ceylan (D), Hakan Polat, Member, IEEE, \\ and Ozan Keysan (iD) Member, IEEE,
}

\begin{abstract}
Analysis of the velocity skin effect (VSE) in electromagnetic launchers (EMLs) requires a 3-D transient finite element method, unlike magnetic skin and proximity effects. However, VSE is dominant at high speeds, and this creates convergence problems when moving or deformed mesh physics is used in a transient FEM in the 3-D analysis. Commercial finite element software cannot solve the electromagnetic aspects of such a high-speed application with a transient solver in 3-D. Although 2-D approximations can be used, such an approximation overestimates VSE resistance due to geometry simplifications. In this study, we proposed a novel quasi-transient 3-D FEM model where the air-armature region's conductivity is varied to emulate the high-speed motion of the armature. Results showed that 2D approximation; overestimates the VSE resistance by almost 40\%. The proposed VSE model has been included in the EML model, and simulation results compared for experimental results with different EMLs, EMFY-1 and EMFY-2, and showed good agreement.
\end{abstract}

Index Terms-Velocity skin effect (VSE), electromagnetic launchers (EMLs), finite element method (FEM), transient analysis

\section{INTRODUCTION}

Electromagnetic launchers (EMLs) utilize Lorentz force to accelerate projectiles up to a few thousand $\mathrm{m} / \mathrm{s}$. Although the skin and proximity effects are well-known phenomena in electromechanical systems, the velocity skin effect (VSE) is an EML specific phenomenon that occurs in the rails at the vicinity of the armature as stated in $[1]-[3]$. To observe the VSE on the current distribution, transient finite element method (FEM) simulations are required to calculate the magnetic field distribution at the armature and rail interface, which is quite complicated to compute [4]. Moreover, due to large armature velocity, transient 3-D FEM has a convergence problem due to interpolation errors between stationary and moving nodes. Thus, special FEM codes are required since commercial software is only used for static mesh elements in simulations due to their formulations [4].

N. Tosun, D. Ceylan, H. Polat and O. Keysan are with the Department of Electrical and Electronics Engineering, Middle East Technical University, 06800 Ankara, Turkey.

(c) 2020 IEEE. Personal use of this material is permitted. Permission from IEEE must be obtained for all other uses, in any current or future media, including reprinting/republishing this material for advertising or promotional purposes, creating new collective works, for resale or redistribution to servers or lists, or reuse of any copyrighted component of this work in other works. Under review.

Corresponding author: Ozan Keysan e-mail: keysan@metu.edu.tr
EMAP3D code uses Lagrangian description and rail reverse motion techniques [5], which is also called the upwinding method to simulate an electrical sliding contact problem under transient conditions. EFFE software [6], [7] is also have the same technique, rail moves backward rather than the armature movement. Quasi-static, time-harmonic FEM are also used to model velocity induced currents [8], [9].

Transient FEM simulations that have an armature movement have two computational domains; stationary and moving. Discontinuity in the field distributions at the intersection of the domains is handled using the continuity conditions. However, this is not the only strategy. Quasi-Eulerian methods, in which the material properties are swept in the direction of the motion instead of mesh movement, can also be used. Shatoff et al. [10] moved the rail conductivity backward to simulate the armature relative motion, and current distribution at the armature is targeted. Hundertmark et al. [11] used time and position-dependent conductivity function in the airarmature region to simulate the transients due to the armature movement. In the previous study [12], we obtained a lumped VSE resistance through a similar conductivity function to enhance the simulation model's accuracy and verified with experiments.

In this work, we take a step further to studies about quasitransient FEM in the literature. The quasi-transient model is used to calculate the accuracy of the 2-D models in VSE calculations. VSE resistances are obtained for armature speeds. The study is conducted within a 2-D quasi-transient FEM and verified with 2-D transient FEM, where the armature move as a domain. Then, the VSE resistance is calculated in 3-D with quasi-transient FEM. The fitted VSE resistances are added to the 3-D static FEM simulations like in [12], [13] individually as a figure of merit. The constructed model is compared with experimental results to evaluate the performances of VSE models. Both EMFY-1 and EMFY-2 launchers, which are shown in Fig. 1 and Fig. 2, respectively, are used in this study. The geometric parameters of those launchers are given in Table 1.

In Section II, the procedure to obtain VSE resistance and the simulation methods are explained. In Section III, a 3-D hybrid simulation model that uses fitted VSE resistances to examine their performances is explained. In Section IV, the simulation results and experimental data are compared, and the accuracy of 2-D approximation in VSE analysis is discussed. 
TABLE I

GEOMETRIC PARAMETERS OF LAUNCHERS

\begin{tabular}{lll} 
& EMFY-1 & EMFY-2 \\
\hline rail thickness & $20 \mathrm{~mm}$ & $60 \mathrm{~mm}$ \\
rail height & $25 \mathrm{~mm}$ & $60 \mathrm{~mm}$ \\
rail seperation & $25 \mathrm{~mm}$ & $50 \mathrm{~mm}$ \\
rail height & $3 \mathrm{~m}$ & $3 \mathrm{~m}$ \\
\hline
\end{tabular}

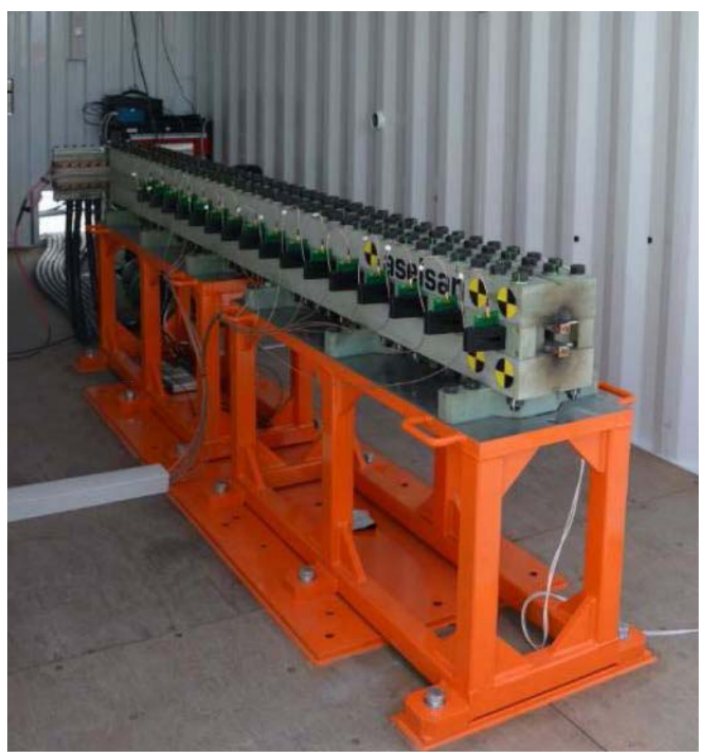

Fig. 1. EMFY-1 EML [14].

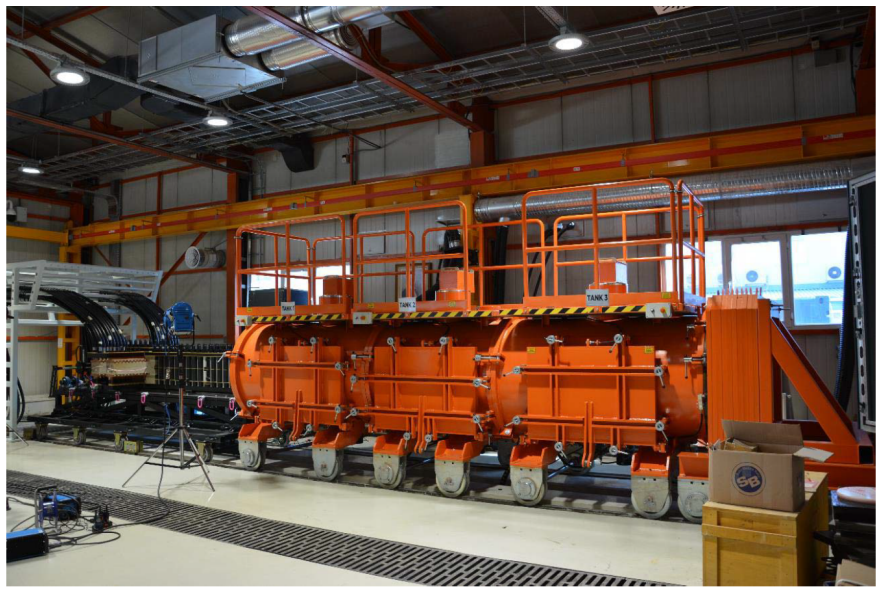

Fig. 2. EMFY-2 EML [15].

\section{Methodology}

The block diagram of the conducted work is presented in Fig. 3. Three different simulation models are developed, which will be explained in the following subsections in detail. The main aspects of the simulation models are given in Table II In the analysis part, velocity-dependent VSE resistances are fitted with given velocity samples $v$, using two different models; 2-D transient and 3-D quasi-transient FEM. The fitted resistances are added to 3-D hybrid FEM, excited with PPS. The purpose of the hybrid model is to increase the overall simulationtest accuracy [13]. The described model will be explained in the following subsections. In this study, the hybrid model's objective is to increase simulation-experiment accuracy and create a figure of merit that is used to compare performances of VSE resistances obtained by 2-D and 3-D analysis.

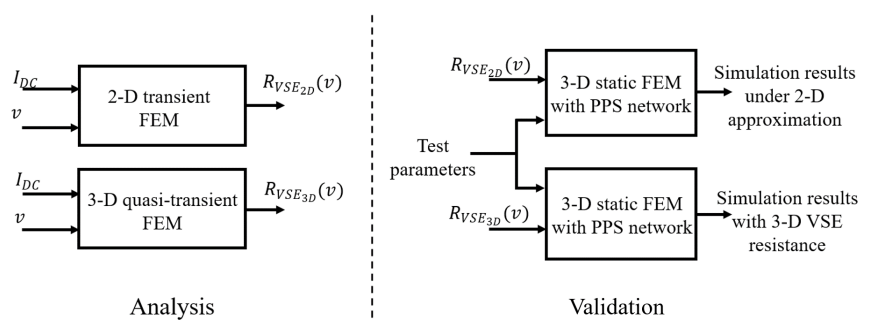

Fig. 3. The methodology used to investigate the accuracy of 2-D approximation on VSE resistance.

\section{A. Analysis}

Developing a realistic simulation model is critical for both the design and tactical operations of the EML. However, since the launcher operates in extreme conditions both electrically and mechanically, simulations are challenging. In this section, two different simulation models that are used in the analysis part are explained. 2-D transient FEM and 3-D quasi-transient models are compared in terms of the calculated VSE resistance obtained from various armature speeds. In 2D Transient FEM Model, the armature is modeled with moving mesh elements. Therefore, there are discontinuous mesh nodes in the railarmature interface different from static models. These mesh nodes are represented in Fig. 4a. The continuity condition is applied in the interface to interpolate the field variables. Magnetic insulation is settled as the continuity condition. In Fig. 5, the current distribution in rails and the armature are given for steady and moving armatures. The current clustering phenomenon is observed in the 2-D transient simulation model. The procedure used for modeling VSE resistance has two parts: Firstly, 2-D transient FEM simulations are made at various constant speeds where DC excitation is used. Therefore, only DC and VSE resistances are calculated. Secondly, The evaluated VSE resistance is separated into two parts; transient and steady parts, which are used for fitting functions [13].

Proper use of the velocity feature in the electromagnetic analysis needs deep physical insight. When the moving domain is of bounded extent in the direction of the motion, material properties vary in this direction [16]. It should be noted that the mesh movement at the sliding contact is not an option in 3-D. The underlying issue is that curl elements, which the interface uses for the magnetic vector potential being solved, give significant interpolation errors if the mesh is discontinuous. Thus, in the 3-D quasi-transient FEM, the armature mesh elements are not moved or deformed. However, the air-armature region's electrical conductivity is updated with time in the armature motion direction to emulate the movement. This approach can be called an Eulerian method since the material properties are modified to simulate the movement [10]. It should be noted that since the mesh elements are simply-connected, field variables are continuous in all boundaries; thus, there is no need to introduce continuity condition. 
TABLE II

PROPERTIES OF SIMULATIONS MODELS USED IN THIS STUDY.

\begin{tabular}{|c|c|c|c|}
\hline Model Name & Armature Mesh Type & Armature Motion & Purpose \\
\hline 2-D Transient FEM & moving & moving meshes & obtaining $R_{\text {VSE } \text { under 2-D assumption. }}$ \\
3-D Quasi-Transient FEM & static & conductivity sweep & obtaining $R_{\text {VSE without 2-D approximation. }}$ \\
3-D Hybrid FEM & static & the armature has no motion. & comparison with experimental results. \\
\hline
\end{tabular}
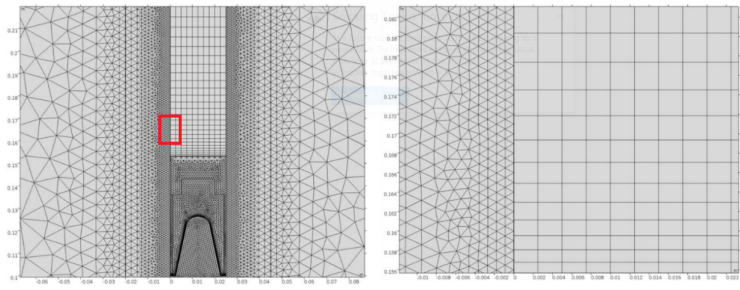

(a) Discontinuous mesh nodes.
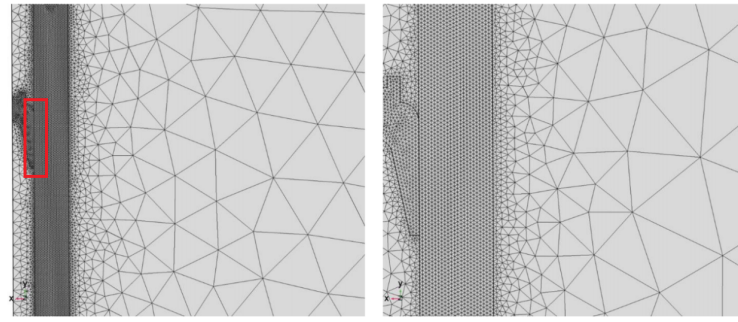

(b) Continuous mesh nodes.

Fig. 4. Mesh structures of the simulation models. 2-D transient FEM model uses discontinuous mesh structure (a). However, 3-D quasi-transient FEM and 3-D Hybrid FEM models use continuous mesh structure (b) since they use static armature. Red rectangles represent the zoom areas.
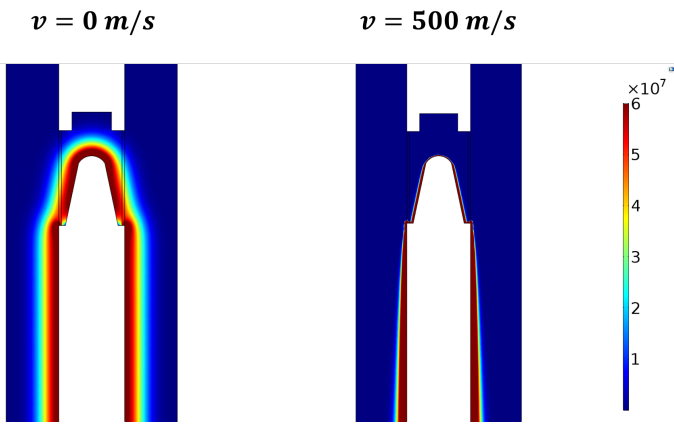

Fig. 5. 2-D transient FEM results with stationary and moving armature.

In Fig. 7, the current distribution in the rail, which is calculated from 2-D transient, and 2-D quasi-transient models, are given. It shows that the current distribution in the quasitransient method is almost the same as the transient method in 2-D. The time and position-dependent conductivity function is given in Fig. 7. The armature is modeled as a rectangular prism for simplicity. Thus, the only variation in the electrical conductivity in the direction of the armature movement.

Since all simulation models are time-dependent, a proper time discretization is required. Variable-step solvers modify the step size during the simulation. They decrease the step size to increase accuracy when a model's states change quickly and increase the step size to avoid taking unnecessary steps when the model's states are changing gradually. Thus, fixed-

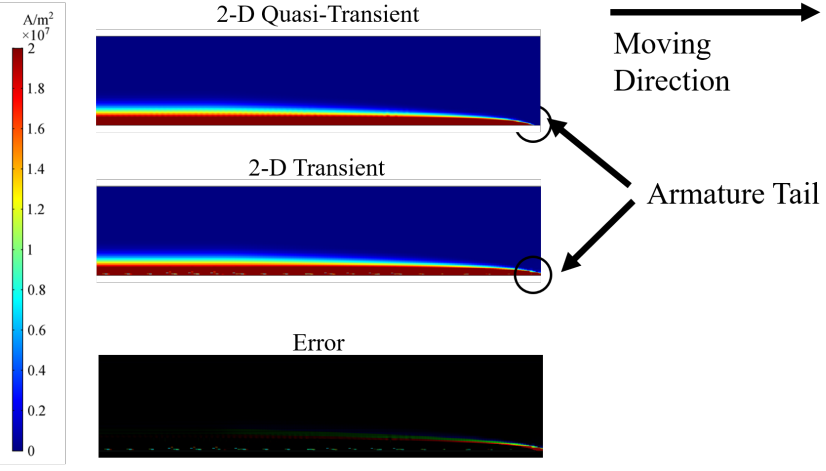

(a) $v=500 \mathrm{~m} / \mathrm{s}$

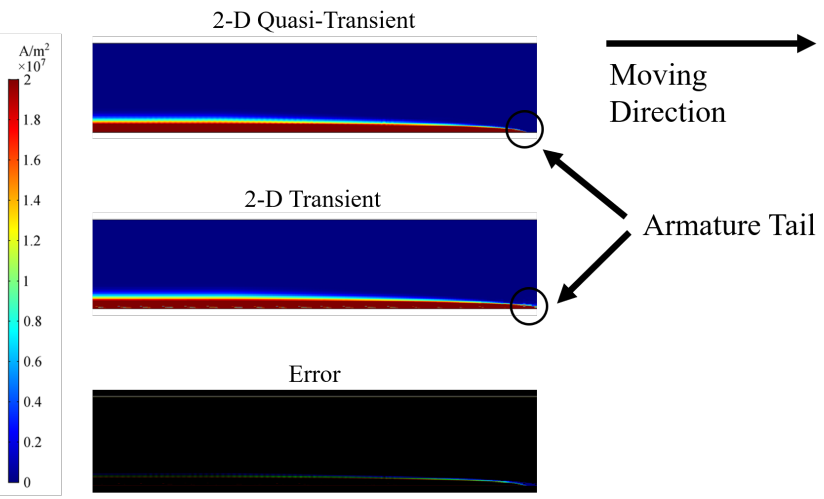

(b) $v=1000 \mathrm{~m} / \mathrm{s}$

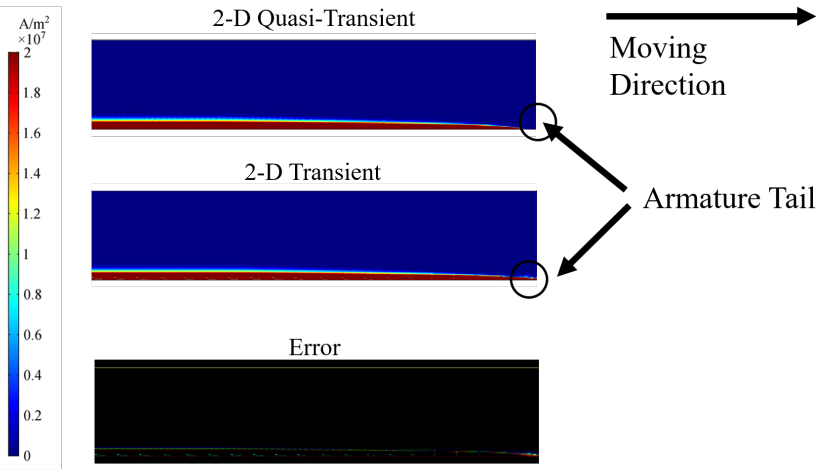

(c) $v=2000 \mathrm{~m} / \mathrm{s}$

Fig. 6. Comparisons of 2-D quasi-transient model and 2-D transient model in terms of rail current distribution at $v=500 \mathrm{~m} / \mathrm{s}, v=1000 \mathrm{~m} / \mathrm{s}$ and $v=2000 \mathrm{~m} / \mathrm{s}$. Error distributions of current densities are also demonstrated. The points that the rail current enters the armature (armature tails) and the armature's moving direction are indicated with arrows. In error plots, zero errors are indicated with black pixels. 
step solvers have longer simulation times, but they are on the safe side. In this study, we used a variable-step solver with a minimum time-step penalty. Time-dependent solver convergence settled a value calculated from armature velocity to ensure the sudden conductivity jump. For example, for $1 \mathrm{~mm}$ maximum mesh size in the air-armature domain and $1000 \mathrm{~m} / \mathrm{s}$ armature speed, time-dependent solver convergence is allowed to have 0.5 us step size as maximum to prevent discontinuity due to movement. Such convergence limitation increases simulation time, but it enables us to see the transient due to the armature movement.

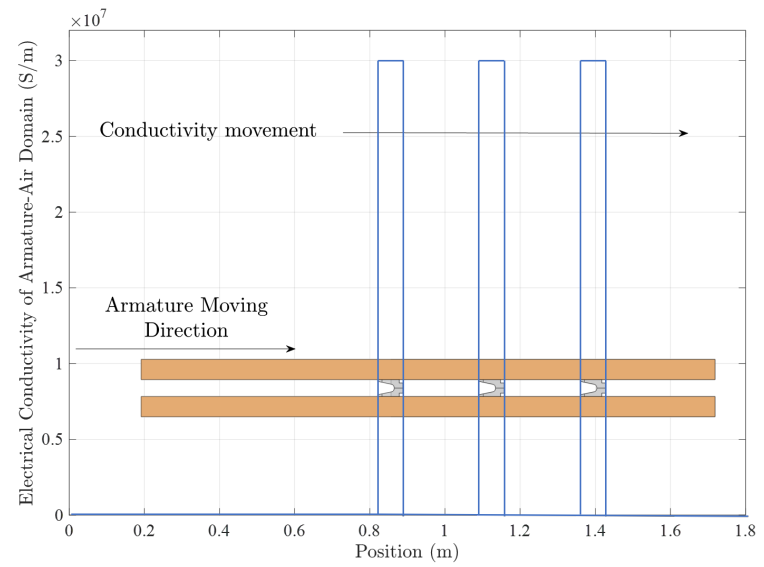

Fig. 7. Time and position dependent conductivity function used in the armature-air region.

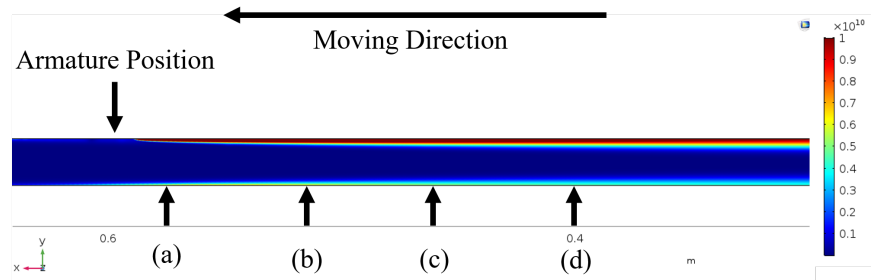

Fig. 8. The current distribution in the middle surface of the rail when the armature has $1200 \mathrm{~m} / \mathrm{s}$ speed. The armature position and the moving direction are shown using arrows. Moreover, small letters are used to mark positions of cross-sections, which are used to show current distributions in Fig. 9

2-D models are built on the fact that field variables do not change in the symmetry direction. Thus, in the VSE investigation of EMLs, it means the rails' current distribution does not change through rail height. However, the validity of the assumption is not determined in the literature. In Fig. 8 , the current distribution in the middle surface of the rail when the armature has $1200 \mathrm{~m} / \mathrm{s}$ speed is shown. Arrows denoted with letters used to show cross-section positions are used in Fig. 9. The rail's middle surface is demonstrated with black lines in the rail cross-sections in the same figure. An important argument that can be extracted from Fig. 9 is the current distribution is not homogeneous through rail height, which also sure signs the error comes from the 2-D approximation of $\mathrm{R}_{\mathrm{vse}}$. The current distribution, which is calculated from 2-D transient/quasi-transient FEM models, is similar to the current
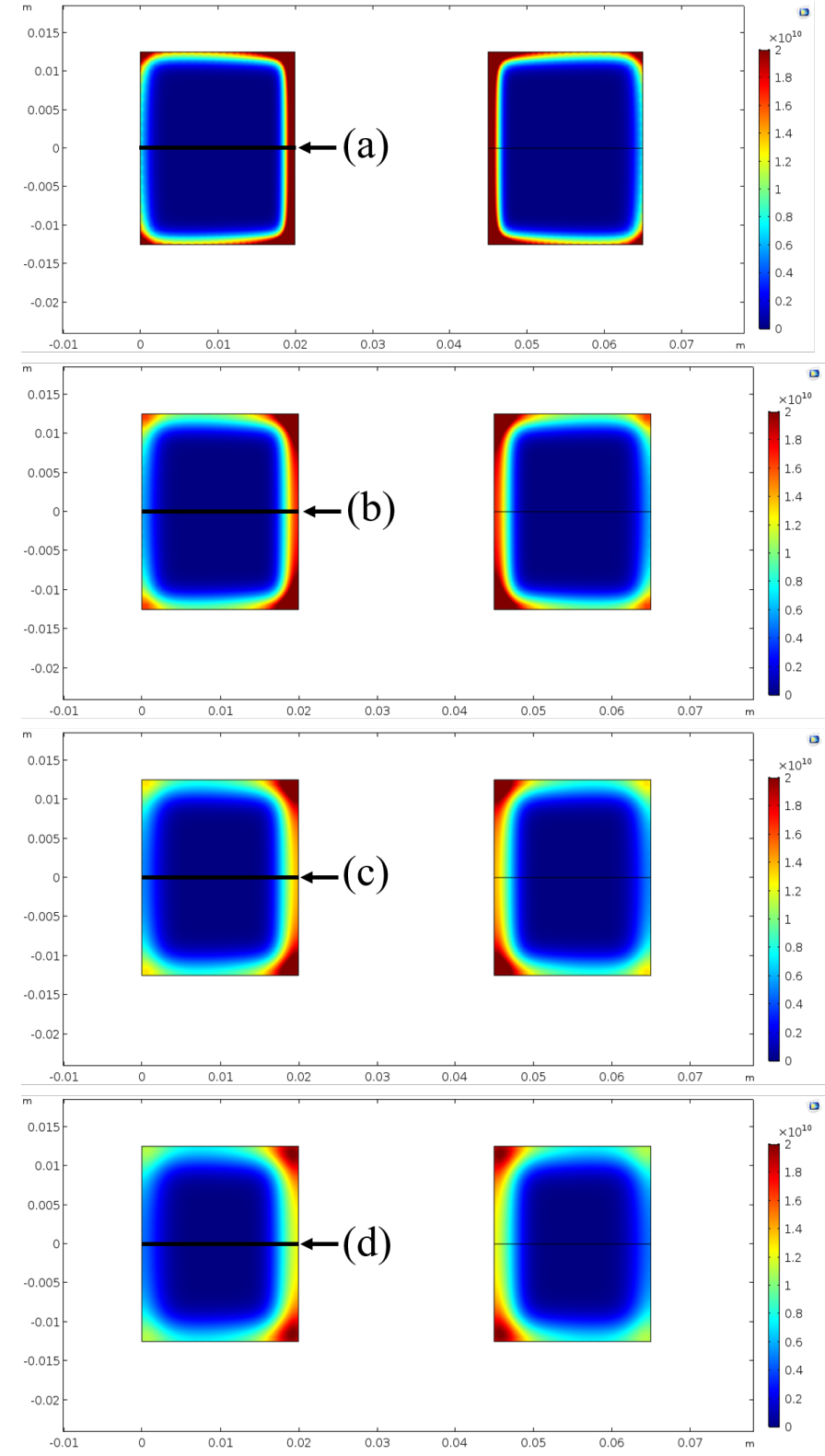

Fig. 9. Current distribution in rails with various rail cross-sections. 3-D quasitransient FEM analysis showed that the current narrowing phenomena due to VSE are more dominant in the middle of the rail than other regions. 2-D approximation modeled the system like it is infinitely high and identical; therefore, the VSE effect is considered a uniform across to rails. This result causes overestimation of $R_{\mathrm{VSE}}$ when the 2-D approximation is applied.

distribution at the rail's middle surface only. The outcomes obtained from the analysis part are listed as follows.

1) The verification of the quasi-transient model is made in 2-D. The current distributions as well as the resistance calculations are identical.

2) The current clustering phenomenon due to existence of VSE occurs mostly the middle surface of the rails.

\section{B. Validation}

The validation section's primary goal is to find a figure of merit for VSE resistance calculation methods. For that purpose, the fitted VSE resistances from the previous section 
are embedded in the 3D-static FEM coupled with PPS circuitry, which is called the hybrid FEM model. Then, several experiments are conducted to explore the precision of the simulations. Before going into the comparison study results, a brief explanation of the hybrid FEM model is given.

EMLs have a high aspect ratio due to long rails, resulting in a large number of mesh elements. In order to reduce computational load, partial rail modeling can be used. The rail portions which are not model in the FEM model can be modeled as lumped circuit parameters. Ceylan et al. [13] used a hybrid model for simulation of EMFY-1 EML. One of the geometries which we used in the 3-D hybrid FEM model is shown in Fig. 10 This geometry belongs to the EMFY1 launcher, which has a $25 \times 25 \mathrm{~mm}$ caliber. The armature is modeled stationary at pre-load distance, $0.7 \mathrm{~m}$. The structure of the hybrid model, which we used, is demonstrated in Fig. 11.

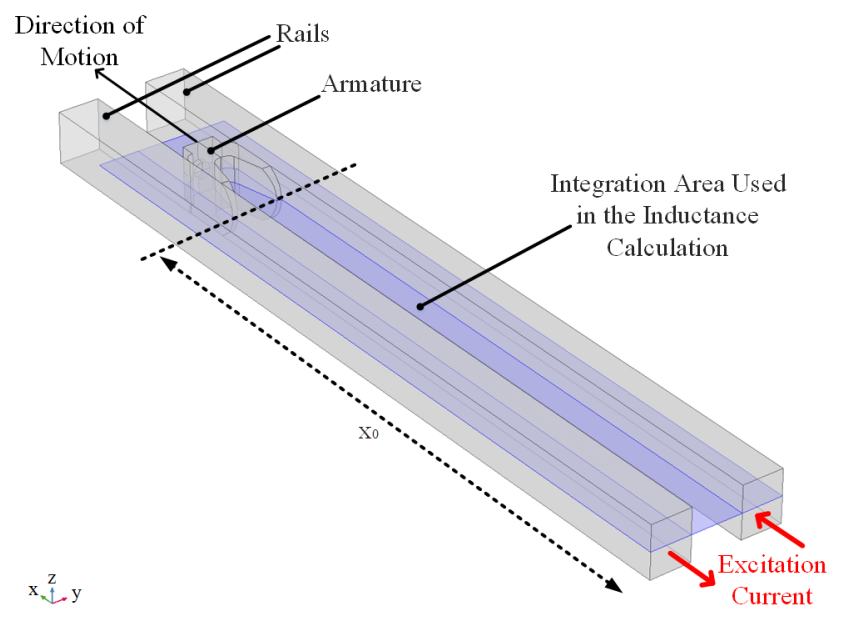

Fig. 10. The geometry used in 3-D FEM analysis for EMFY-1. The integration area which used in the inductance calculation is shown as purple. The area between rails is called exterior boundary and the inductance, which is related in this area called $\mathrm{L}_{\text {ext }}$. The area inside the rail is called the interior boundary. The Interior boundary is used in rail inductance calculation, which is called $\mathrm{L}_{\text {int }}$.

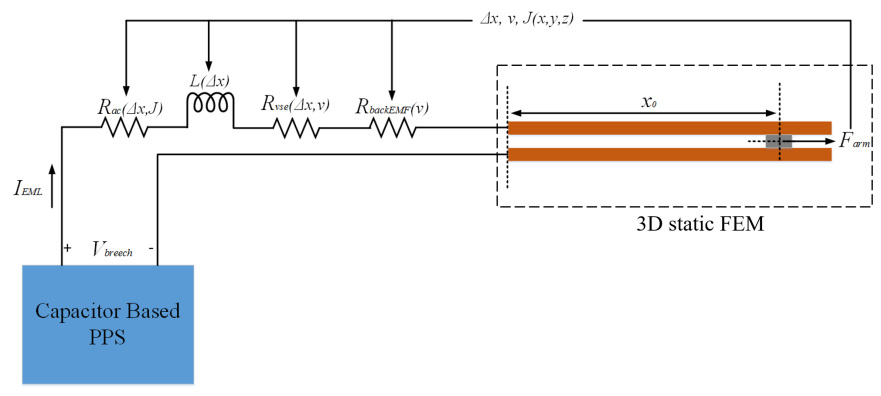

Fig. 11. The hybrid FEM model used to simulate EMLs. Resistances and inductances between the PPS network and 3-D FEM body use to model the armature movement. With that structure, not only the computational complexity is reduced, but also the total number of mesh number is reduced as all the rail length is not required to be meshed.

It should be noted that the hybrid FEM model has a stationary armature. Therefore, the current distribution of the rails did not cluster due to VSE. The current distributions in the rail when 3-D static and 3-D quasi-transient methods are used are illustrated in Fig. 12. The physical effects such as inductance and resistance variations due to armature movement added to the simulation environment using lumped parameters. One of the mentioned physical effects is VSE resistance, which is denoted as $R_{v s e}$, which is calculated from 2-D transient and 3-D quasi-transient analysis.

Other lumped parameters which we used to model the rail portion which related with armature regions are defined as exterior inductance, $\mathrm{L}_{\text {ext }}$ interior inductance $\mathrm{L}_{\mathrm{int}}$, AC resistance $R_{\mathrm{ac}}$ and back EMF resistance $R_{\text {emf }}$. $L_{\text {ext }}$ is used to model inductance contribution in the air region between rails. However, $L_{\text {int }}$ is used to model inductance contribution in the rails and air region between rails, excluding the exterior region. Total inductance contribution due to $p$ amount armature movement can be modeled, to sum up, that inductance (1).

$$
L(t)=p\left(L_{\text {int }}^{\prime}(t)+L_{\text {ext }}^{\prime}(t)\right)
$$

Back EMF resistance, $R_{\text {backEMF }}$ formulation can be extracted from (2) to (5). EML can be considered as single turn coil. Therefore, linkage flux $\lambda(\mathrm{t})$ can be calculated from the inductance and rail current $\mathrm{I}(\mathrm{t})$.

$$
\begin{aligned}
& \lambda(t)=L(t) I(t) \\
& \mathcal{E}=\frac{d \lambda(t)}{d t}=\frac{d L(t)}{d t} I(t)+L(t) \frac{d I(t)}{d t} \\
& \mathcal{E}=R_{\text {backEMF }} I(t)+L(t) \frac{d I(t)}{d t}
\end{aligned}
$$

Back EMF due to armature movement is modeled as a resistance. Although it behaves as a voltage source, this assumption, from a mathematical perspective, does not pose a problem. $R_{a c}$ can be calculated using the joule loss in the rail portion volume $V$. In (6), the resistivity of the rails is denoted as $\rho_{\text {rail }} . \mathrm{J}_{\text {rail }}$ and $\|$.$\| represents the current density inside the$ rails and $\mathrm{l}_{2}$ norm, respectively.

$$
R_{a c}=p \frac{1}{I(t)^{2}} \iiint_{V} \rho_{\text {rail }}\left\|J_{\text {rail }(t)}\right\|^{2} d V
$$

The only lumped parameter which can not be computed directly from the hybrid FEM model is $\mathrm{R}_{\mathrm{vse}}$. This parameter is imported from the studies which were done with 2-D transient and 3-D quasi-transient methods. Since all the other parameters are kept constant, the error that comes from $R_{v s e}$ calculations is directly transferred to the outputs of the hybrid FEM simulations.

\section{RESULTS}

In Fig. 13. VSE resistance is obtained by the 2-D transient FEM model for various speeds is demonstrated. The result shows that both transient and steady VSE resistance levels increase with the armature speed as expected. $R_{v s e}$ values, 


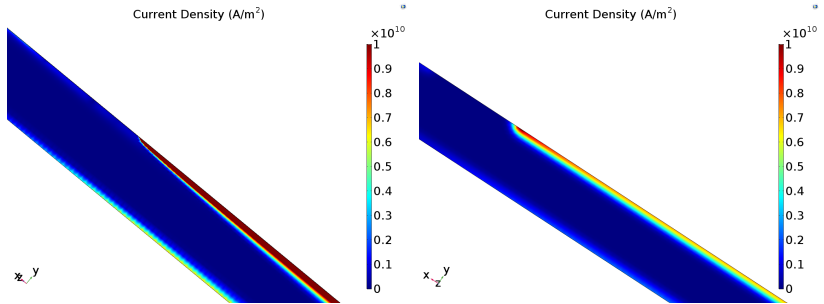

Fig. 12. The current distribution $\left(\mathrm{A} / \mathrm{m}^{2}\right)$ in rail 3-D quasi-transient simulation (left) and static armature simulation (right). Quasi-transient analysis made with armature which has $1000 \mathrm{~m} / \mathrm{s}$ velocity.

which are obtained by 3-D quasi-transient simulations are illustrated in Fig. 14. After exploiting lumped parameters including $\mathrm{R}_{\mathrm{vse}}$, which obtained 3-D quasi-transient and 2-D transient FEM results, the hybrid simulation model is tested with experimental results to investigate the differences. The rail currents are compared to evaluate accuracy of $R_{v s e}$ fitting, since it is a significant contributor [13] to total EML resistance.

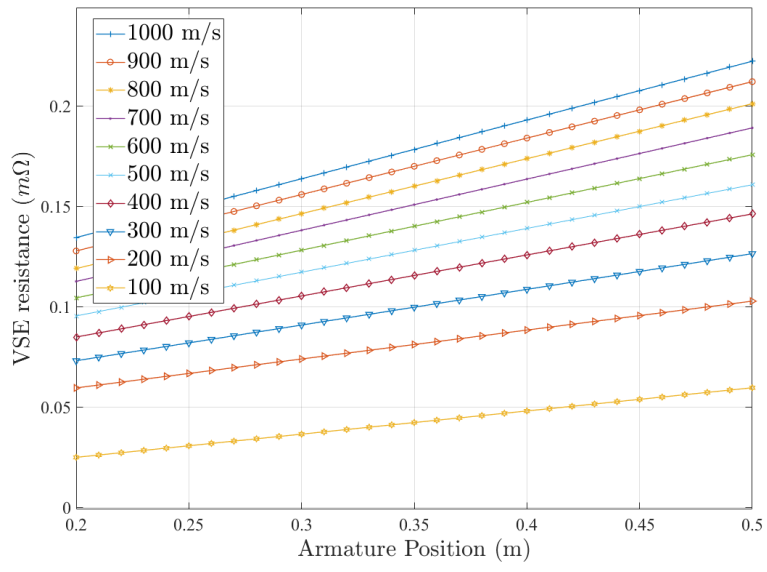

Fig. 13. 2-D transient simulation results for VSE resistance with respect to armature position graph for armature velocities from $100 \mathrm{~m} / \mathrm{s}$ to $1000 \mathrm{~m} / \mathrm{s}$.

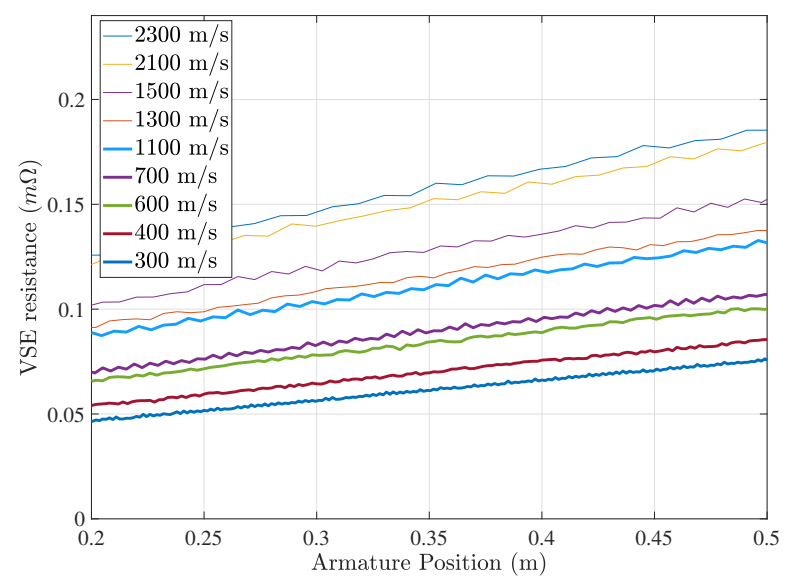

Fig. 14. 3-D quasi-transient simulation results for VSE resistance with respect to armature position graph for armature velocities from $300 \mathrm{~m} / \mathrm{s}$ to $2300 \mathrm{~m} / \mathrm{s}$.
The open area launch tests of EMFY-1 were conducted in 2018 [13]. EMFY-2 was tested indoors in 2019. Experiment parameters of EMFY-1 and EMFY-2 are given in Table 3, respectively.

The results of the 3-D Hybrid FEM simulations where 3$\mathrm{D}$ quasi-transient and 2-D transient methods used for $\mathrm{R}_{\mathrm{vse}}$ as well as experimental results are compared in Fig. 15-18. Results show that the 3-D quasi-transient method is the most consistent with experimental results.

TABLE III

PARAMETERS OF EMFY-1 AND EMFY-2 EXPERIMENTS

\begin{tabular}{lllll} 
& \multicolumn{2}{c}{ EMFY-1 } & \multicolumn{2}{c}{ EMFY-2 } \\
$2-34-5$ & Exp. A & Exp. B & Exp. C & Exp D. \\
\hline Initial Electrical Energy & $750 \mathrm{~kJ}$ & $625 \mathrm{~kJ}$ & $1227 \mathrm{~kJ}$ & $3241 \mathrm{~kJ}$ \\
Launch Package Mass & $41.4 \mathrm{~g}$ & $41.6 \mathrm{~g}$ & $932 \mathrm{~g}$ & $1033.2 \mathrm{~g}$ \\
Capacitor Voltage & $3560 \mathrm{~V}$ & $3250 \mathrm{~V}$ & $4000 \mathrm{~V}$ & $6500 \mathrm{~V}$ \\
\hline
\end{tabular}

The overestimation of the $\mathrm{R}_{\mathrm{vse}}$ with the 2-D method can be explained using the experimental results. At the later stages of the launching process, the EML circuit can be modeled as a passive RL circuit. The reason is the fact that after the discharging of the last PPS unit, all free-wheeling diodes, $\mathrm{D}_{1}$ to $\mathrm{D}_{\mathrm{N}}$, gets into conduction mode. Thus, capacitors are disconnected from the EML, as in Fig. 19. The rail current decay rate depends only on the EML inductance, $\mathrm{L}_{\mathrm{EML}}$, and resistance, $R_{E M L}$. The 2-D transient simulations have faster decay than experimental results due to the overestimation of $R_{\text {EML }}$. It should be noted that discrepancy at the late stage of the experiments links up with $\mathrm{R}_{\mathrm{vse}}$ dominance at the late stage of the launch [13].

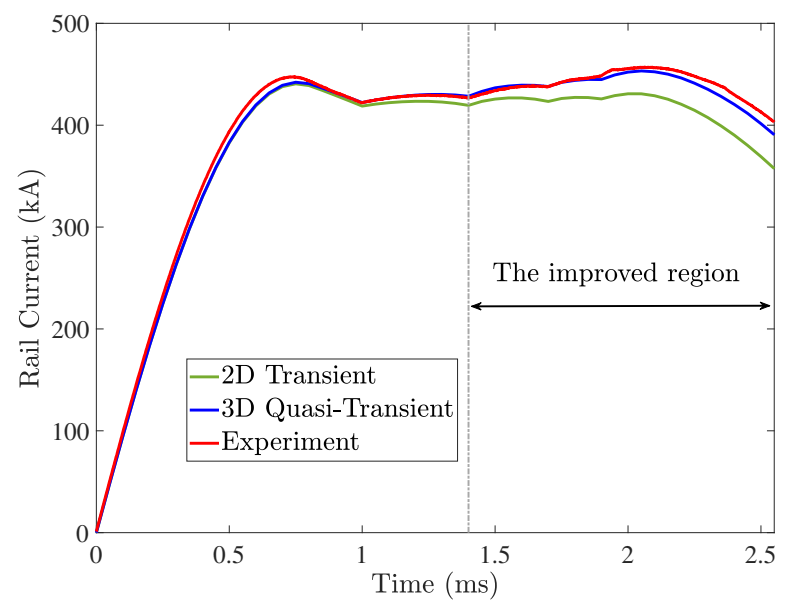

Fig. 15. Simulation and experimental results for the rail current of the experiment-A with EMFY-1

\section{DISCUSSION}

Although the quasi-transient method can model velocitydependent resistance calculation in EMLs, there are some limitations of the proposed method. One of the disadvantages of the quasi-transient method is that the armature can not be analyzed in detail due to geometry limitation due to the conductivity function definition. In this study, the armature 


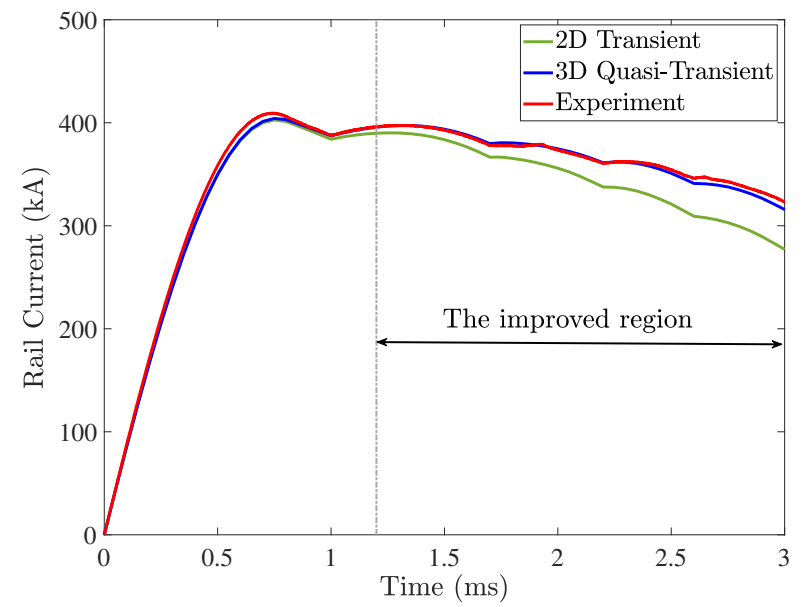

Fig. 16. Simulation and experimental results for the rail current of the experiment-B with EMFY-1

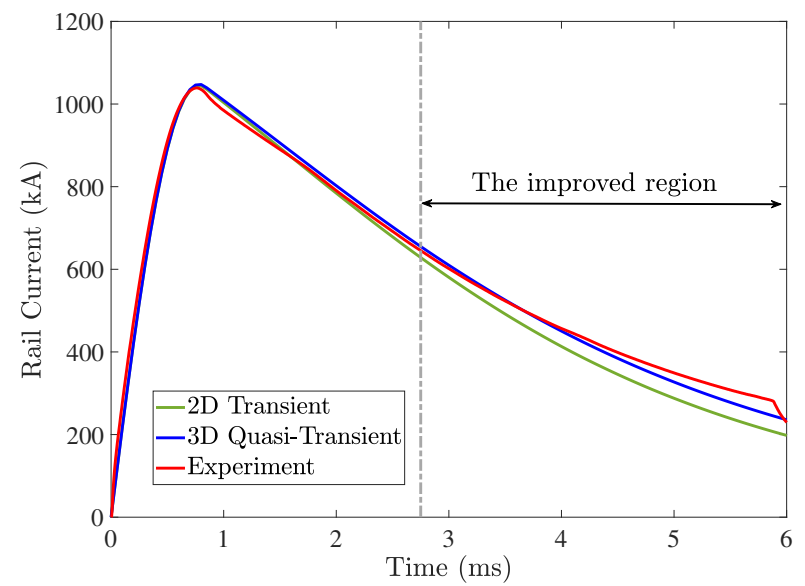

Fig. 17. Simulation and experimental results for the rail current of the experiment-C with EMFY-2

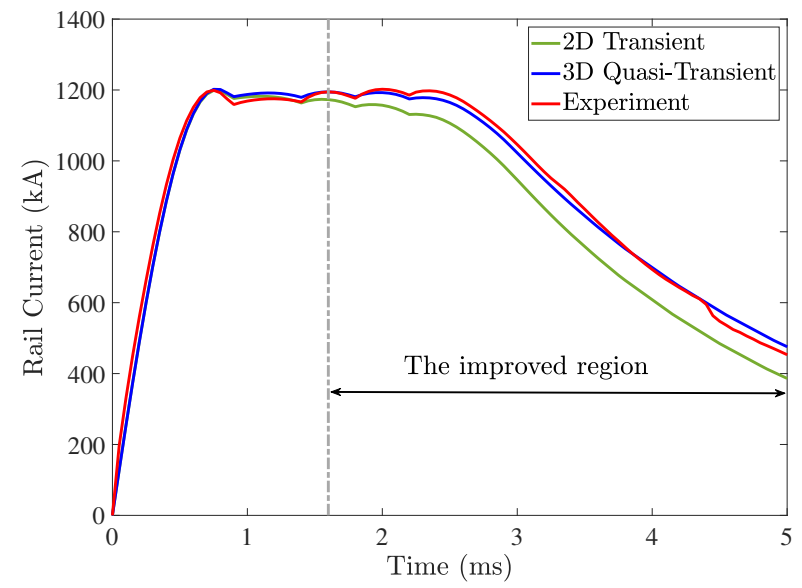

Fig. 18. Simulation and experimental results for the rail current of the experiment-D with EMFY-2

is modeled as a rectangular prism, as shown in Fig. 20, but

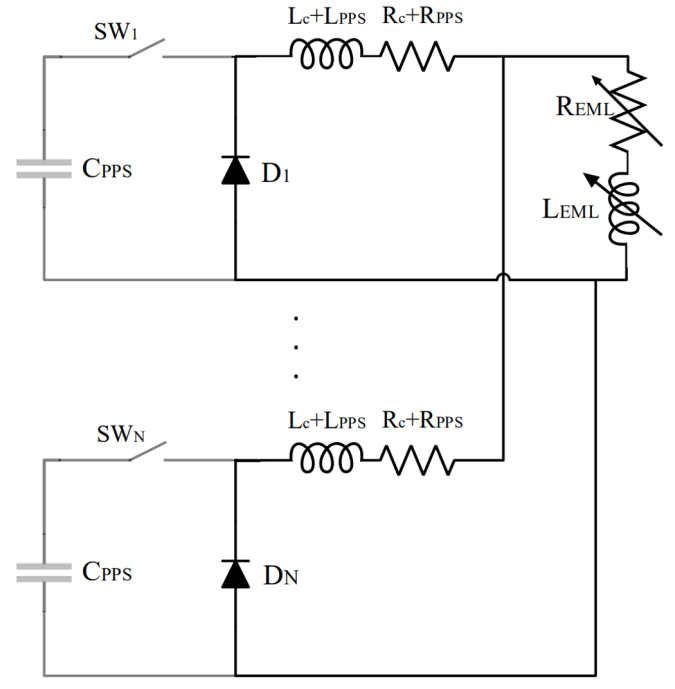

Fig. 19. The circuit schematic of the last stage of the launch. Disconnected units are demonstrated using gray color. $R_{P P C}$ and $L_{P P C}$ represent the PPS resistance and inductance, respectively. $R_{c}$ and $L_{c}$ represent cable resistances and inductances.

they have more complex geometries in actual cases. Another disadvantage is that the time-dependent solver convergence is limited when the quasi-transient method is used. To prevent sudden conductivity jumps between mesh elements, time-steps are locked at a specific value, which increases the simulation time.

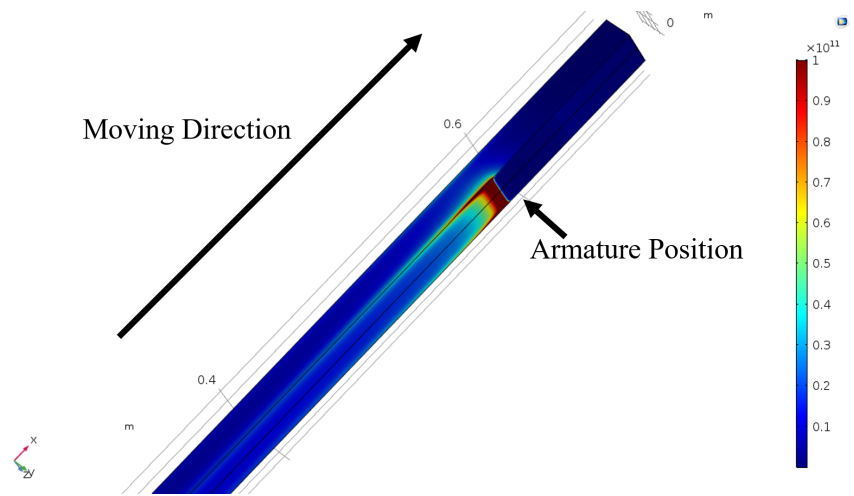

Fig. 20. The current density distribution $\left(\mathrm{A} / \mathrm{mm}^{2}\right)$ in the rail with 3-D quasi-transient model. The armature modelled as rectangular prism.

\section{CONClusion}

In this study, a comparison study of modeling VSE with 2-D transient and 3-D quasi-transient FEM is presented. The Eulerian method is used to model the armature movement in the rails in the 3-D transient model. VSE resistance models are used in the hybrid FEM-lumped model to compare the models' accuracy with the experiments. The following remarks are obtained.

1) The quasi-transient method can be used model VSE in commercial FEM codes. 2-D transient and quasitransient FEM give identical current distribution in the rails. 
2) The hybrid FEM model simplifies the computational problems of EMLs, such as the number of mesh elements and the requirement of the armature motion in the simulation. Although the field distribution at the sliding contact does not contain the current narrowing phenomenon, the VSE's resistive effects are added to the simulation environment, which improves losses and efficiency calculations.

3) The 2-D model overestimates VSE resistance. The reason for that is the current narrowing phenomena occur in the middle of the rail more dominantly than the up and downs side. 2-D approximation assumes that the current distribution is identical in all rail height direction. However, this is not the case in the actual rail current distribution.

The exploited VSE resistance is a critical lumped parameter for the overall system simulations. The simulation-experiment coherence is enhanced with the proposed method. The same procedure is applicable with different caliber EMLs or actuators, which has VSE on their conductors.

\section{ACKNOWLEGMENT}

The authors would like to thank Mustafa Karagöz, Baran Yıldırım, and Ibrahim Güngen for their support and collaboration.

\section{REFERENCES}

[1] T. G. Engel, J. M. Neri, and M. J. Veracka, "The velocity and efficiency limiting effects of magnetic diffusion in railgun sliding contacts," in 2008 14th Symposium on Electromagnetic Launch Technology, June 2008, pp. $1-5$.

[2] — "Characterization of the velocity skin effect in the surface layer of a railgun sliding contact," IEEE Transactions on Magnetics, vol. 44, no. 7, pp. 1837-1844, July 2008.

[3] G. C. Long and W. F. Weldon, "Limits to the velocity of solid armatures in railguns," IEEE Transactions on Magnetics, vol. 25, no. 1, pp. $347-$ 352, Jan 1989.

[4] S. Tan, J. Lu, B. Li, Y. Zhang, and Y. Jiang, "A new finite-element method to deal with motion problem of electromagnetic rail launcher," IEEE Transactions on Plasma Science, vol. 45, no. 7, pp. 1374-1379, July 2017.

[5] K. Hsieh, "A lagrangian formulation for mechanically, thermally coupled electromagnetic diffusive processes with moving conductors," IEEE Transactions on Magnetics, vol. 31, no. 1, pp. 604-609, Jan 1995.

[6] S. An, B. Lee, Y. Bae, Y. Lee, and S. Kim, "Numerical analysis of the transient inductance gradient of electromagnetic launcher using 2-d and 3-d finite-element methods," IEEE Transactions on Plasma Science, vol. 45 , no. 7, pp. 1635-1638, July 2017.

[7] — "Numerical analysis on the transient inductance gradient of the resistive overlay rail on the sliding electrical contact," IEEE Transactions on Plasma Science, vol. 47, no. 5, pp. 2339-2342, May 2019.

[8] O. Liebfried, M. Schneider, T. Stankevič, S. Balevičius, and N. Žurauskiené, "Velocity-induced current profiles inside the rails of an electric launcher," IEEE Transactions on Plasma Science, vol. 41, no. 5, pp. 1520-1525, May 2013.

[9] T. Stankevič, M. Schneider, and S. Balevičius, "Magnetic diffusion inside the rails of an electromagnetic launcher: Experimental and numerical studies," IEEE Transactions on Plasma Science, vol. 41, no. 10, pp. 2790-2795, Oct 2013.

[10] H. Shatoff, D. A. Pearson, and A. E. Kull, "Simulation of dynamic armature motion in a railgun with coupling of electromagnetic, thermal and structural effects using shifted finite element fields," in 2005 IEEE Pulsed Power Conference, 2005, pp. 253-256.

[12] N. Tosun, H. Polat, D. Ceylan, M. Karagöz, B. Yildirim, Güngen, and O. Keysan, "A hybrid simulation model for electromagnetic launchers including the transient inductance and electromotive force," IEEE Transactions on Plasma Science, pp. 1-9, 2020.

[11] S. Hundertmark and M. Roch, "Transient 3-d simulation of an experimental railgun using finite element methods," in 2012 16th International Symposium on Electromagnetic Launch Technology, 2012, pp. 1-5.
[13] D. Ceylan, M. Karagöz, Y. Çevik, B. Yıldırım, H. Polat, and O. Keysan, "Simulations and experiments of emfy-1 electromagnetic launcher," IEEE Transactions on Plasma Science, vol. 47, no. 7, pp. 3336-3343, July 2019.

[14] M. Karagoz, Y. Çevik, E. Tan, A. Civil, O. Cavbozar, U. Gocmen, B. Yildirim, E. Durna, and M. S. Sahin, "Aselsan emfy-1 electromagnetic launcher: First experiments," in 2017 IEEE 21st International Conference on Pulsed Power (PPC), 2017, pp. 1-3.

[15] M. Karagoz, A. Civil, B. Yildirim, E. B. Yurdakul, E. Durna, E. Tan, O. Cavbozar, U. Gocmen, and Y. Cevik, "Aselsan electromagnetic launch laboratory: First shot," IEEE Transactions on Plasma Science, pp. 1-6, 2020.

[16] "Comsol multiphysics $\AA$ ac/dc module user's guide v. 5.4. comsol ab, stockholm, sweden.” pp. 226-227, 2018

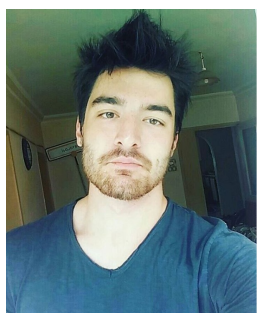

Nail Tosun received the B.Sc. degree from the Department of Electrical and Electronics Engineering, Middle East Technical University (METU), Ankara, Turkey, in 2019, where he is currently pursuing the M.Sc. degree. His current research interests include electromagnetic FEM analysis, optimization of electrical machines and power electronics.

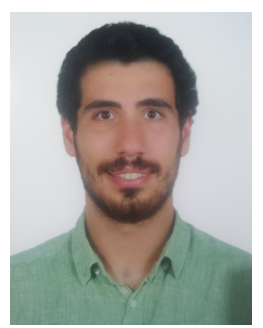

Doğa Ceylan received the B.Sc. and M.Sc. degrees from the Department of Electrical and Electronics Engineering, Middle East Technical University (METU), Ankara, Turkey, in 2016 and 2018, respectively. He is currently working toward the Ph.D. degree with the Electromechanics and Power Electronics Group at the Eindhoven University of Technology, the Netherlands. His current research interest focuses on design and optimization of electrical machines, electric vehicles, and electromagnetic launchers.

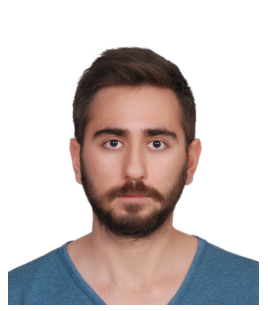

Hakan Polat received the B.Sc. degree from the Department of Electrical and Electronics Engineering, Middle East Technical University (METU), Ankara, Turkey, in 2018, where he is currently pursuing the M.Sc. degree. His current research interests include, electromagnetic launchers, pulsed-power sources, renewable energy and wireless power transfer.

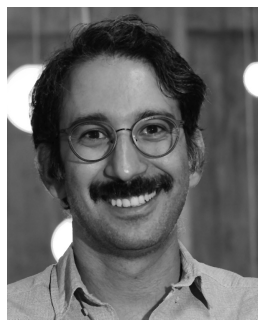

Ozan Keysan received the master's degree from Middle East Technical University (METU), Ankara, Turkey, in 2008, and the Ph.D. degree from the University of Edinburgh, Edinburgh, Scotland, in 2014. He is currently an Assistant Professor with the Electrical and Electronics Engineering Department, METU. His current research interests include renewable energy, design and optimization of electrical machines, smart grids, superconducting machines, and permanent-magnet machines. 